\title{
Robotic Assisted Radio-Frequency Ablation of Liver Tumors - Randomized Patient Study
}

\author{
A. Patriciu, M. Awad, S.B. Solomon, M. Choti, D. Mazilu, \\ L. Kavoussi, and D. Stoianovici
}

The Johns Hopkins University, Baltimore MD 21224

\begin{abstract}
The minimally invasive treatment of liver tumors represents an alternative to the open surgery approach. Radio-frequency ablation destroys a tumor by delivering radio-frequency energy through a needle probe. Traditionally, the probe is placed manually using imaging feedback. New approaches use robotic devices to accurately place the instrument at the target. The authors developed an image-guided robotic system for percutaneous interventions using computed tomography. The paper presents a randomized patient study comparing the manual versus robotic needle placement for radio-frequency ablation procedures of liver tumors. The results of this study show that in our case robotic interventions were a very viable solution. Several treatment parameters such as radiation exposures and procedure-times were found to be significantly improved in the robotic case.
\end{abstract}

\section{Introduction}

Minimally invasive image guided procedures are increasingly popular due to their potential benefits such as reduced trauma and improved recovery time. In such procedures an instrument, usually a needle, is percutaneously placed to an anatomical target under image guidance. The imaging methods used for guidance include all types of imaging: ultrasound, X-Ray, computed tomography (CT), and magnetic resonance imaging (MRI). In the traditional approach the needle is manually placed by the physician. This requires a significant amount of training, hand-eye coordination, 2D to 3D extrapolation skills, and in the same time it can deliver a large amount of radiation to the patient and medical personnel if imaging uses X-Rays. To overcome these problems researchers proposed a number of needle guides, shields and even robotic manipulators. Robots have the advantage of operating in the digital space of the image, potentially have better manipulation performance, and are insensitive to radiation.

Robot manipulators for minimally invasive image guided interventions have been developed starting in the late 80 's. Several robotic systems have been purposely developed for CT-guided interventions. A system named Minerva was designed for stereotactic neurosurgery at the Micro-engineering Laboratory of the Swiss Federal Institute of Technology Center 3. Masamune et al. developed a minimally invasive surgical system for neurosurgery [6]. An MRI compatible needle driver was designed by the same group using ultrasonic motors and nonferromagnetic materials [7]. 
In recent years a growing effort was devoted to building robots that can work with magnetic resonance imaging (MRI). Chinzei et al. 1] developed an MR compatible robot that can work in an open magnet MR. Kaiser et al. developed a system for breast biopsy [4. The system uses ultrasonic motors for actuation and a combination of laser range sensors and custom built optical rotation code transducers for position feedback.

Another device for MR guided interventions was developed by Krieger et al. [5] at the Johns Hopkins University. The manipulator was designed for transrectal prostate interventions. The position of the device in MR coordinates is computed using special design position coils. The system was used initially on animal studies and after the initial validation was redesigned and used on a patient pilot study.

The validation of a surgical system requires model studies, followed by cadaver or animal studies before the system can be clinically used. In order for a system to demonstrate an improvement over a traditional approach, it is commonly evaluated in a randomized patient study. The procedures outcome variables are compared for the robotic assisted and manual approaches. Despite the relatively large number of experimental surgical robotic systems, there are very few randomized patient studies that assess their functionality in real clinical environments. Cleary et al. 2] reported a randomized clinical study with twenty patients. The study compared the outcome of a joystick controlled robotic needle placement versus manual needle placement. The study showed that the robot can be at least as accurate as the human operator. Even though in the reported study the system did not include computer controlled image guidance, the study provided important validation methodologies for surgical systems in the interventional suite.

This paper reports the results of a randomized patient study comparing the robotic assisted versus manual needle placement. Both cases are performed under CT-guidance. The goal of the study is to evaluate wether or not the robotic system can improve the time, radiation exposure, and/or accuracy of the RF procedure.

\section{Materials and Method}

The interventional system comprises a surgical robot [10 attached to a CTScanner mobile table. The target is defined by the surgeon/radiologist in $\mathrm{CT}$ image space. In order to compute the position of the target in robot space it is necessary to compute first the transformation between the CT image space and the robot space - the registration transformation. This is computed using the laser system provided with the CT-scanner [8]. A short description of the surgical robot and of the registration technique is presented below.

\subsection{Surgical Robot}

The surgical robot (Figure 1) presents a bridge like structure comprising a XYZ cartesian stage and a PAKY-RCM robotic module connected through a $6 \mathrm{DOF}$ 


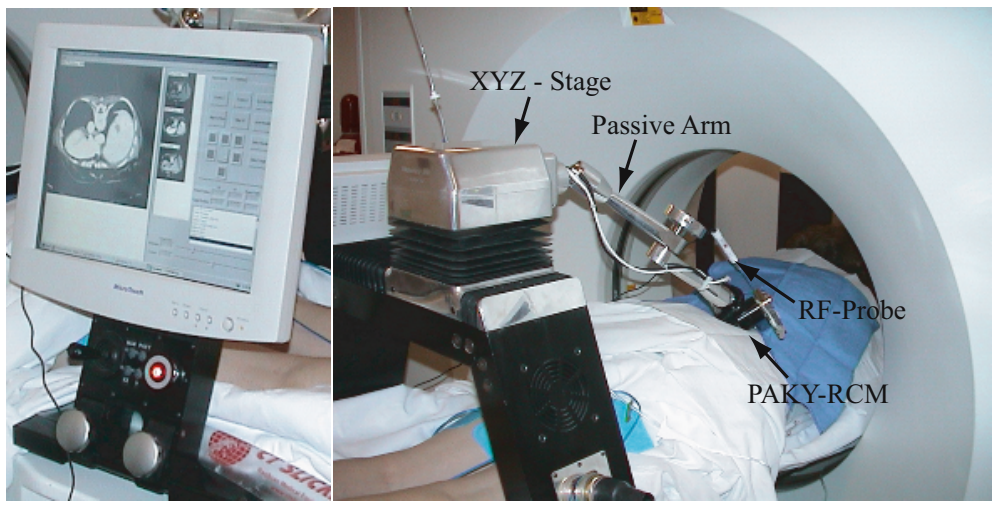

a)

b)

Fig. 1. AcuBot robot: a) User interface detail; b) Acubot in a robotic assisted CT guided RF Ablation

passive arm [10]. The RCM (Remote Center of Motion) module is capable of precisely orienting an instrument (needle) around a fixed point distal to the mechanism. PAKY (Percutaneous Access to the KidneY) is a needle driver allowing for the needle insertion to be performed after alignment with the target. The instrument is loaded initially with its point at the fulcrum. The PAKY-RCM ensemble is initially positioned using the passive arm such that the fulcrum is close to the desired entry point. The XYZ cartesian stage can be used for small adjustments in the initial robot positioning until the point of the needle is at the skin entry site.

The user interface includes an LCD mounted on the bridge adapter (Fig. 11) together with a joystick and an emergency stop button. The manipulator is controlled using an industrial PC fitted with a PCX-DSP Motion Engineering card. The manipulator can be attached to a CT table as well as an OR table, using special adapters.

\subsection{CT-Registration and Targeting}

The registration procedure involves two main steps, as follows:

Step 1. This step defines the current image plane $\left(L P_{1}\right)$ in the robot coordinate system by using the laser alignment process (Fig. 2). The current image plane is defined in robot coordinates by placing the instrument/needle in two different positions contained in that plane. The robot is initially placed such that the needle point is in the image plane, then the instrument is rotated around its tip and placed in two different positions $\vec{\nu}_{i}, i=1,2$ contained in the image plane. In the current implementation, the containment condition is verified visually by the operator observing if the laser marker shines the end of the needle. In future implementations an optical sensor will be attached to the needle end for automatic plane detection. The cross product of $\vec{\nu}_{1} \times \vec{\nu}_{2}$ defines the $\vec{z}$-axis of the CT-Scanner in robot space. At this stage, the 


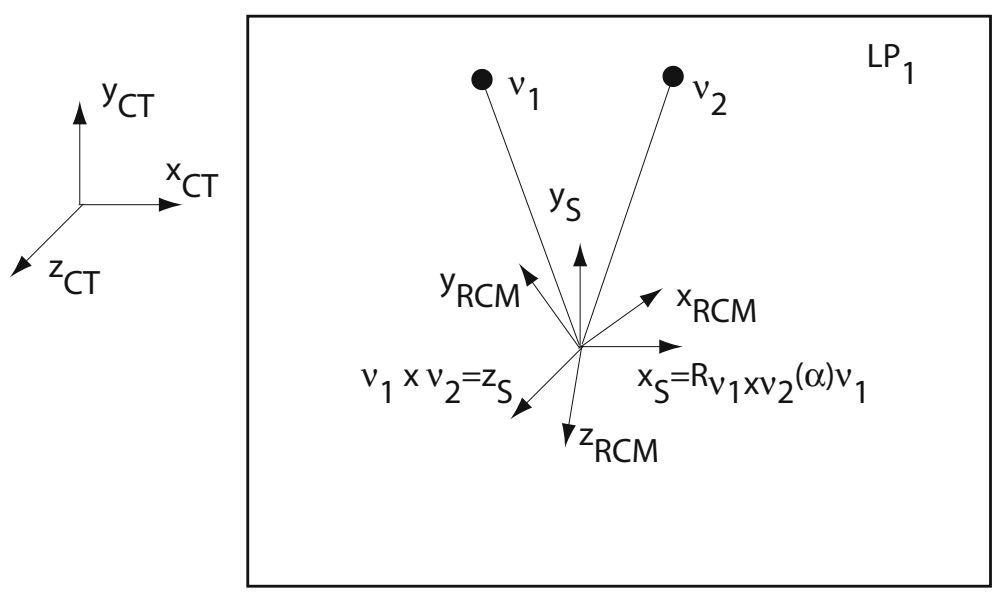

Fig. 2. Registration method and associated coordinates frames. $x y z_{R C M}-$ RCM robot coordinate frame; $x y z_{C T}$ - CT coordinate frame; $x y z_{s}$ - auxiliary coordinate frame, parallel with $x y z_{C T}$ and with the same origin as $x y z_{R C M}$

robot can be restricted to move in the $L P_{1}$ image plane. This could be used to remotely manipulate the needle in the image space in a similar way that radiologists presently perform CT fluoroscopy manual interventions.

Step 2. The remaining registration data is image-based and uses the image acquired for entry-point/target specification. An image is acquired at the $\vec{\nu}_{1}$ needle orientation. The angle between the image of the needle and the $\vec{x}$-axis of the CT is $\alpha$. Then, the CT $\vec{x}$-axis in robot coordinates is $\operatorname{Rot}_{\nu_{1} \times \nu_{2}}(\alpha) \nu_{1}$, where $\operatorname{Rot}_{\delta}(\theta)$ is a rotation matrix about the axis $\delta$ with the angle $\theta$. This completes the necessary rotational registration data. The translational component is computed using the current position in the image of the tip of the needle which is also the origin of the robot space and the data stored in the DICOM image.

The physician selects the target in an intra-operative CT image displayed on the monitor of the robot. The image coordinates are transformed to robot coordinates using the registration transformation. The coordinates of the target are then used by the robot controller to accurately align and insert the needle at the specified location, if commanded by the physician.

\subsection{Randomized Patient Study}

The system accuracy and reliability were initially tested in a preclinical environment. The mean accuracy recorded over $n=25$ trials was $1.7 \mathrm{~mm}$ with a standard deviation of 0.8 9. While the preclinical study represents a good engineering validation of the system, a clinically usable system is more demanding; it is necessary to prove that the system improves the results of a real procedure. After the system obtained the authorization of the hospital, the performance of the system was objectively assessed using a randomized study involving fourteen 
patients undergoing radio-frequency ablation of the liver tumors. The patients were randomized to undergo the robot assisted RF probe placement or conventional CT guided manual probe placement.

For the manual needle placement the following steps were performed:

1. The patients were placed on the CT table. A volume scan was initially acquired to localize the lesion and plan the procedure. The entry site was cleaned with betadine; local lidocaine was administered over the planned entry site.

2. The needle was manually inserted at the desired location under CT fluoroscopy guidance. During the insertion the patient is instructed by the physician to hold his breath.

3. The radio-frequency ablation was performed.

In the robotic needle placement the following steps were performed:

1. The patients were placed on the CT table. A volume scan was initially acquired to localize the lesion and plan the procedure. The entry site was cleaned with betadine; local lidocaine was administered over the planned entry site.

2. The robot was placed such that the point of the needle entry point was at the planned entry point. The registration procedure was performed.

3. A CT image is acquired such that the target is contained in that image.

4. The robot automatically oriented the instrument.

5. The radiologist manually inserted the needle in with the amount specified by the targeting algorithm under patient breath-hold; the direction of the needle is maintained by the robot. The optimal approach would be to automatically insert the needle using the needle driver. In the current setting this is not possible due to a plastic insulation coating present on the RF needle barrel.

Table 1. Recorded treatment variables for $\mathrm{CT}$ guided RF ablation randomized patient study

\begin{tabular}{|c|c|c|}
\hline$\#$ & Treatment Variable & Description \\
\hline 1 & number of probe passes & $\begin{array}{r}\text { How many times was the RF probe } \\
\text { placement adjusted. }\end{array}$ \\
\hline 2 & time to successful targeting & $\begin{array}{r}\text { Time in minutes from the moment when } \\
\text { the CT image used to define the target was } \\
\text { acquired and the moment when } \\
\text { the probe was at the desired location. }\end{array}$ \\
\hline 3 & overall procedure time & $\begin{array}{c}\text { The total duration of the procedure, } \\
\text { including the RF teatment, measured in minutes. }\end{array}$ \\
\hline 4 & patient radiation exposure & mrem \\
\hline 5 & physician radiation exposure & mrem \\
\hline 6 & complications & complications during or after the procedure \\
\hline 7 & ablation completeness & did the treatment cover the entire tumor? \\
\hline
\end{tabular}


6. The radio-frequency ablation of the tumor is performed after a verification of the needle placement accuracy.

For all patients, the treatment variables presented in Table 1 were recorded.

The accuracy of the procedure was characterized by the number of probe passes required to reach a satisfactory instrument placement. The radiation exposure of the patient was measured using a radiation badge placed in the proximity of the entry site. The radiation exposure of the physician was measured at the hand level using a radiation monitoring ring badge. The study was designed to evaluate the potential of the robot to reduce the procedure costs by reducing the overall procedure time, as well as, and the potential of the robot to reduce the radiation exposure of the patient and physician.

\section{Results}

The study shows that the number of passes to reach the target is lower in the robotic case $(p=0.0006)$. Also, the robotics approach delivers a smaller amount of radiation to the physician $(p=0.0004)$ and to the patient $(p=0.0007)$. The time to reach the target $(p=0.0001)$ and the overall procedure time $(p=$ $0.00005)$ were lower in the case of robotic approach when compared to the manual case. All statistical tests were performed using Student's t-Test. Table 2 presents the mean values and standard deviations of the treatment variables measured. Furthermore, all ablative procedures were well tolerated in all patients with no difference in the ability to achieve complete ablation $(>90 \%)$ in the two groups. $\mathrm{RF}$ ablation was considered successful if no local recurrence was detected by $\mathrm{CT}$ or MRI after 6 months of follow-up imaging (either CT or MRI). For all patients in both robotic and manual groups, no local recurrence was detected.

Table 2. Data statistics for the CT guided robotic versus manual RF ablations patient study

\begin{tabular}{|c|c|c|c|c|c|c|c|c|}
\hline & \multicolumn{3}{|c|}{ Robotic } & \multicolumn{5}{c|}{ Manual } \\
\cline { 2 - 10 } & Mean & Std. Dev. & Max & Min & Mean & Std. Dev. & Max & Min \\
\hline $\begin{array}{c}\text { time to successful } \\
\text { targeting (min) }\end{array}$ & 3.57 & 1.13 & 4 & 2 & 8.57 & 1.99 & 12 & 6 \\
\hline $\begin{array}{c}\text { overall procedure } \\
\text { time(min) }\end{array}$ & 44.57 & 6.68 & 53 & 36 & 67.57 & 8.28 & 57 & 78 \\
\hline $\begin{array}{c}\text { number of probe } \\
\text { passes }\end{array}$ & 1 & & 1 & 1 & 3.71 & 1.25 & 6 & 2 \\
\hline $\begin{array}{c}\text { patient radiation } \\
\text { exposure (mrem) }\end{array}$ & 469.71 & 177.09 & 836 & 279 & 7075.71 & 3181.65 & 2923 & 12522 \\
\hline $\begin{array}{c}\text { physician radiation } \\
\text { exposure (mrem) }\end{array}$ & 0 & & & & 577.57 & 250.56 & 327 & 1097 \\
\hline
\end{tabular}




\section{Conclusion and Discussion}

The paper presents a randomized study designed to assess the performances of robotic assisted CT guided RF-ablations procedures. The robotic assisted radio-frequency ablation was compared against the standard manual approach through several treatment variables. The study showed that the robotic assisted treatment of liver tumors is feasible, and it provides an improvement in terms of the procedure time, procedure accuracy, physician radiation exposure and patient radiation exposure.

Robotic assisted approaches present the potential to reduce costs by reducing the time of the procedure. The needle placement accuracy influences the outcome of the procedure; precise needle placement ensures that the tumor is destroyed with more reliable margins, while minimizing the healthy tissue damage. The reduction of radiation exposure is equally advantageous for patient and physician. Since there is a maximum amount of radiation that a human can tolerate the reduction in radiation exposure translates in the physician's ability to perform more procedures annually. The results of this study show that the robotic approach can be beneficial for CT-guided RF ablations procedures.

The proposed testing methodology can be used to validate the real performances of other robotic systems designed for minimally invasive procedures. Future developments will evaluate its potential application to other CT guided interventions.

\section{Disclosure}

"Under licensing agreements between ImageGuide and the Johns Hopkins University, the authors are entitled to a share of royalty received by the University on ImageGuide's sales of products embodying the robotic technology described in this article. Under a private license agreement, authors are entitled to royalties on ImageGuide's sales of products embodying the technology described in this article. The authors and the University own Image Guide stock, which is subject to certain restrictions under University policy. Dr. Stoianovici is a paid consultant to Image Guide and a paid member of the company's Scientific Advisory Board. Dr. Stoianovici's participation in the study was limited to technical maintenance of the robot. Dr. Stoianovici did not interact with patients and was not involved in clinical data analysis. The terms of this arrangement are being managed by the Johns Hopkins University in accordance with its conflict of interest policies."

\section{Acknowledgements}

This work was partially supported by grant No. CA88232 from the National Cancer Institute (NCI) and by the American Foundation of Urologic Disease (AFUD/AUER) Research Scholar Program and Dornier MedTech. Its contents are solely the responsibility of the authors and do not necessarily represent the official views of NCI and AFUD. 


\section{References}

1. K. Chinzei, N. Hata, F. Jolesz, and R. Kikinis. Mr compatible surgical assist robot: System integration and preliminary feasibility study. In Proceedings of Third International Conference On Medical Robotics, Imaging and Computer Assisted Surgery, pages 921-930, Pittsburgh, 2000.

2. K. Cleary, V. Watson, D. Lindisch, A. Patriciu, D. Mazilu, and D. Stoianovici. Robotically assisted interventions: Clinical trial for spinal blocks. In Medical Image Computing and Computer-Assisted Intervention - Miccai 2003, Pt 2, volume 2879 of Lecture Notes in Computer Science, pages 963-964. 2003.

3. D. Glauser, H. Fankhauser, M. Epitaux, J.-L. Hefti, and A. Jaccottet. Neurosurgical robot minerva, first results and current developments. In Proc. 2nd Int. Symp. on Medical Robotics and Computer Assisted Surgery, pages 24-29, Baltimore, Maryland, 1995. MRCAS '95 Symposium, C/O Center for Orthop Res, Shadyside Hospital, Pittsburgh, Pa.

4. W. A. Kaiser, H. Fischer, J. Vagner, and M. Selig. Robotic system for biopsy and therapy of breast lesions in a high-field whole-body magnetic resonance tomography unit. J. Investigative Radiology, 35(8):513-519, 2000.

5. A. Krieger, R.C. Susil, C. Menard, J.A. Coleman, G. Fichtinger, E. Atalar, and L.L. Whitcomb. Design of a novel mri compatible manipulator for image guided prostate interventions. Biomedical Engineering, IEEE Transactions on, 52(2):306313, 2005. TY - JOUR.

6. K. Masamune, L. H. Ji, M. Suzuki, T. Dohi, H. Iseki, and K. Takakura. A newly developed stereotactic robot with detachable drive for neurosurgery. In Medical Image Computing and Computer-Assisted Intervention - Miccai'98, volume 1496 of Lecture Notes in Computer Science, pages 215-222. 1998. Times Cited: 2 Article.

7. K. Masamune, E. Kobayashi, Y. Masutani, M. Suzuki, T. Dohi, H. Iseki, and K. Takakura. Development of an mri-compatible needle insertion manipulator for stereotactic neurosurgery. Journal of Image Guided Surgery, 1:242-248, 1995.

8. Alexandru Patriciu, Stephen Solomon, Louis R. Kavoussi, and Dan Stoianovici. Robotic kidney and spine percutaneous procedures using a new laser-based ct registration method. In WJ Niessen and M.A. Viergever, editors, Medical Image Computing and Computer-Assisted Intervention, volume 2208 of Lecture Notes in Computer Science, pages 249-258, Utrecht, Netherlands, 2001. Springer-Verlag.

9. S. B. Solomon, A. Patriciu, M. E. Bohlman, L. R. Kavoussi, and D. Stoianovici. Robotically driven interventions: A method of using ct fluoroscopy without radiation exposure to the physician. Radiology, 225(1):277-282, 2002.

10. D. Stoianovici, K. Cleary, A. Patriciu, D. Mazilu, A. Stanimir, N. Craciunoiu, V. Watson, and L. R. Kavoussi. Acubot: A robot for radiological interventions. IEEE Transactions on Robotics and Automation, 19(5):927-930, 2003. Times Cited: 0 Article. 